\title{
Introducing the Goal-Question-Metric approach to telecommunications software development: the PITA experiment
}

\author{
V. Sylaidis, I. Nanakis, V. Kopanas \\ INTRACOM S.A. \\ P.O. Box 68, 19002 Peania, Attika, Greece \\ phone: (+30-1) 6860876, fax: (+30-1) 6860887 \\ E-mail : \{vsyl, inan,vkop\}@intranet.gr
}

\begin{abstract}
This paper describes the experience of introducing the Goal-Question-Metric approach for software development process metrication to INTRACOM's Software Design Centre, based on the AMI methodology. This process improvement experiment, named PITA, was supported by EU funds as an ESSI project (proj. no. 21305). The main objective was to provide a comprehensive and relevant frame of measurements to enable software developers to control and eventually improve the processes they employ, thus ensuring consistently high-quality products. The approach involved a formal CMM assessment and derivation /introduction of systematic measurements at project and organisational level, based on goaltree analysis.
\end{abstract}

\section{Keywords}

AMI (Application of Metrics in Industry), CMM (Capability Maturity Model), ESSI (European Systems and Software Initiative), GQM (Goal Question Metric), measurement plan, PIE (Process Improvement Experiment), PITA (Process Improvement Through AMI), Policy Deployment, software metrics, SEI/CMU (Software Engineering Institute at Carnegie-Mellon Univ.), SEPG (Software Engineering Process Group), SPI (Software Process Improvement). 


\section{INTRODUCTION}

In recent years one can observe a large interest and grow in the application of measurement techniques in software project management. This trend can be justified in the context of systematic efforts to overcome the persisting software crisis, which is manifested by phenomena such as budget and schedule overruns, low quality and reliability and in general high cost of quality for software. The situation is compounded by mounting market pressures for ever shorter delivery times of developed software products (Humphrey, 1989; Boehm, 1991; Brooks, 1995).

According to many studies on the application of metrics and models in industrial environments, measurements should be focused on specific goals and be applied to all products, processes and resources throughout the life-cycle. Measurements should be interpreted based on understanding of the organisational context, environment and business goals. This means that measurements must be defined in a top-down fashion, they must be focused and based on goals and models.

In line with the above trends and studies, and in attempting to overcome shortcomings of INTRACOM's existing, rather primitive software development metrics framework, the GoalQuestion-Metric (GQM) approach was introduced in a baseline project through the AMI method. It was meant to support and enhance INTRACOM's software development practices. The introduction of GQM was carried out as a Process Improvement Experiment (PIE) named PITA, in the context of the CEU programme ESSI (European Systems and Software Initiative).

This paper aims at presenting the rationale of the experiment (sec. 1, 2), a brief account of the GQM method and the AMI approach (sec. 3), the objectives and the organisation of the experiment (sec. 4), the current status of experiment activities (sec. 5), lessons learned from introducing GQM (sec. 6) and a set of conclusions and plans for further activities (sec.7). Section 8 finally, provides a basic set of references related to software measurements, GQM and AMI.

\section{THE SOFTWARE DEVELOPMENT PRACTICE INVOLVED}

The PITA experiment introduces the GQM method to INTRACOM's software development practices. The firm produces a wide range of telecommunications and electronics products and systems for the Greek and international markets. Following a pattern which is typical for such firms internationally, in recent years INTRACOM is increasingly involved in embedded and systems software development. The main current software development activity concerns digital telephony. This is performed in the Software Design Centre (SWDC), which hosted the PITA experiment and currently employs close to 200 highly qualified and trained software engineers/designers.

With regard to Software Process Improvement (SPI), activities have been initiated, promoted and coordinated within a formal Policy Deployment scheme (Sheridan, 1993) while at the same time the improvement frame and path of the SEI CMM model has been adopted. 
Prior to the experiment, a basic set of metrics were being used providing a high-level view of the design process and product quality, as well as a basis for bench-marking. However, they did not constitute a set adequately developed and closely linked to actual experiences and problems. Measurements taken within that immature metrics frame had shown some trends but the necessary analysis was insufficient and the results were inconclusive. In addition, the utilised metrics tended to reflect only generic upper management concerns and viewpoints. Thus, metrics usefulness and suitability was often questioned and their acceptance by involved personnel could not be taken for granted, especially when these metrics contradicted everyday experiences. At the same time, there were not adequate measures for monitoring and controlling the progress of improvement actions.

\section{THE GQM APPROACH AND THE AMI METHOD}

Several approaches and experiences related to software measurements are reported in the literature (Grady, 1987; Grady, 1992; Fenton, 1991; Matsubara, 1991; Moeuller, 1992; METKIT, 1992; Kan, 1995). Among them, the Goal-Question-Metric (GQM) approach (Basili, 1994; Gresse, 1995) provides the most mature and well-defined measurement mechanism. This approach has been developed and proposed for application at software development organisations and is based upon the assumption that for an organisation to measure in a purposeful and efficient way, it must first specify the goals for itself and its projects, then it must trace those goals to the data that define and substantiate those goals operationally, and finally provide a framework for interpreting the data with respect to the stated goals. GQM was originally defined for evaluating defects for a set of projects in the NASA Goddard Space Flight Centre environment. Since then, the use of GQM has been expanded to a larger context.

The result of the application of GQM is the specification of a measurement system targeting a particular set of 'issues' and a set of 'rules' for the interpretation of the measurement data. The GQM measurement model has three levels:

1. Conceptual level (GOAL): A goal is defined for an object (product, process, project or resource), for a variety of reasons, with respect to various models of quality, from various points of view, relative to a particular environment.

2. Operational level (QUESTION): A set of questions is used to characterise the way the assessment / achievement of a specific goal will be performed based on some characterising model. Questions try to characterise the object of measurement (product, process, etc.) with respect to a selected quality issue and to determine its quality from a selected viewpoint.

3. Quantitative level (METRIC): A set of data is associated with every question in order to answer it in a quantitative way. The data can be objective (e.g. person hours spent on a task) or subjective (level of user satisfaction).

A GQM model is a hierarchical structure (see Figure 1) starting with a goal, that specifies purpose of measurement, object to be measured, issue to be measured and viewpoint from 
which the measure is taken. The goal is refined in several questions, that usually break down the issue into its major components. Each question is then refined into metrics. The same metric can be used in order to answer different questions under the same goal. Several GQM models can also have questions and metrics in common, provided that when the measure is actually collected, the different viewpoints are taken into account correctly (i.e. the metric might have different values if taken from different viewpoints).

GQM provides a method for top-down metric definition and bottom-up interpretation (see Figure 1). The GQM approach can be used as stand alone for defining a measurement program or, better, within the context of a more general approach to software process improvement.

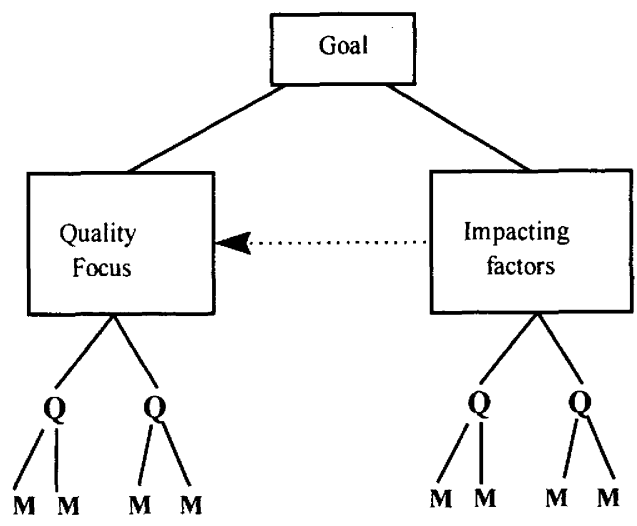

Goal: Measurement Goal

Impacting factors : the key for the ability of analysing reasons for changes and comparing projects

Question: Provides information for the Goal

Metric: Answer to the Question

Figure 1 The GQM model.

The AMI (Application of Metrics in Industry) method (Pulford, 1996) is based on the GQM approach and provides a common-sense framework for quantifying software projects. This framework comprises four principal phases:

1. Assessment of the organisation's environment to define primary goals for measurement.

2. Analysis of the assessment results and primary goals to derive sub-goals and the relevant metrics. The analysis is formalised as a goal tree with a corresponding set of questions to which these metrics are linked.

3. Metrication by implementing a measurement plan and then processing the collected primitive data into measurement data.

4. Improvement as the measurement data are exploited and actions are implemented. Comparison of the measurement data with the goals and questions in the measurement plan will provide guidance towards the achievement of the immediate project goals. When measurements show that a goal has been achieved, there is enough improvement attained to reassess the initial primary goals, i.e. one improvement cycle in the AMI method closes. 
The approach followed by AMI is an adaptation of Deming's widely used Plan-DoCheck-Act cycle for improvement using software measurements. The AMI method is divided in 12 steps with a series of support tools (guidelines, templates and examples), each phase having 3 steps. See Figure 2.

\section{INPUTS}

Enterprise PLANS Business-driven GOALS Environment INFORMATION Past measurement DATA Quality AUDITS

\section{SUPPORT}

SEI Capability Maturity Model

Problem analysis

Examples of primary goals

(1) Assess your environment

(2) Define primary management goals

(3) Check the goals against the assessment

Primary metrication GOALS

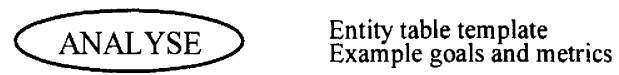

(4) Break-down management goals into sub-goals

(5) Check consistency of resulting goal tree

(6) Identify metrics from sub-goals

\begin{abstract}
Goal tree, questions Metric specifications
\end{abstract}

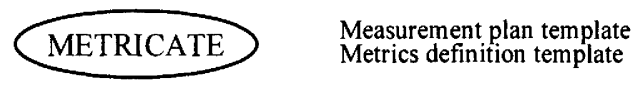

(7) Write and validate measurement plan

(8) Collect primitive data

(9) Verify primary data

Measurement plan Collected data

IMPROVE Guidelines
(11) Validate the metrics
(1) analyse and review measuremt data

(10) Distribute, analyse and review measurement data

(12) Relate the data to goals and implementation actions

Figure 2 The AMI method.

AMI is designed to be flexible enough to employ existing, proven techniques, such as GQM and Software Process Assessment based on SEI's CMM (Carnegie Mellon University, 1995; Dymond, 1995). With the AMI method, the number of metrics that need to be collected is focused on those that correspond to the most important goals. Thus, data collection and analysis costs are limited to the metrics which give the best return. On the other hand, the emphasis on goals and business objectives establishes a clear link to strategic business decisions and helps in the acceptance of measurements by managers, team leaders and engineers.

The AMI method was developed in the context of the AMI project which was funded through the ESPRIT programme to make the technology and techniques of software metrics and measurement available in a simple, straightforward and understandable form that could be easily implemented. The consortium which developed AMI was led by GEC-Marconi and included Alcatel, Bull AG, Objectif Technologie, TECHNOS and South Bank University's Centre for Systems \& Software (CSSE). 


\section{EXPERIMENT OBJECTIVES AND ORGANISATION}

The PITA experiment introduces the Goal-Question-Metric approach via the AMI method and tool to INTRACOM's software development practices. The objectives of PITA experiment can be summarised as follows:

- establishment of a systematic GQM-based framework for software metrics;

- evaluation of the use and effectiveness of AMI approach to support software metrics;

- introduction of a formal assessment (SEI/CMM based) to establish a baseline of software development process maturity and to provide input and guidance for improvements;

- identification of process improvement areas and monitoring/support of corresponding improvement actions;

- dissemination of experience and know-how on AMI and GQM-metrics, both within INTRACOM and its group, as well as in the Greek and international markets;

- continuous monitoring, evaluation and dissemination of results collected from mechanisms established by PITA.

A number of benefits are expected from the implementation of the PITA experiment. These include: enabling and focusing improvements, better support and focused follow-up for scheduled CMM assessments and increased capability in achieving goals and controlling processes. Furthermore, it is expected that PITA experiment will result in better software project planning and control, easier prevention of defects due to requirements and improved product quality. Finally, PITA will contribute to increasing the awareness and motivation of personnel for SPI, as well as providing more opportunities for technology transfer and business partnerships.

In the context of PITA experiment, GQM was integrated as necessary with the selected baseline project. The baseline project for the PITA experiment consists of developing parts of a telecommunications software system for the French market. The project team consisted of 10 persons (including management function) and the project size (effort) was approximately 1,400 person-days. Baseline project selection was based on the availability of new projects, their 'typical' character, and their schedule in relation to the PITA planning. To support the smooth and efficient uptake of AMI method, Objectif Technologie's consulting services were used (training, experience transfer etc.).

As an initial step, setting up of co-ordination and, where required, integration of the experiment with the baseline project was effected. This was done both with the project leader and other key responsible persons, as well as with affected line management and quality engineers. During this step the above personnel was trained in the AMI method. From then on, the basic improvement steps as defined in the AMI method were followed, being implemented at the baseline project. The acquired experience and the confidence built-up for the method allowed similar practices to be gradually taken up in other starting projects as well, even before the experiment was completed.

The four main phases of the PITA experiment, correspond roughly to the 4 phases of the AMI method, supported where possible by the AMI tool.

The first phase in the PITA experiment, involved an assessment, focused mainly on the baseline project. All relevant information from any preceding projects, any special 
restrictions, previously available measurements and audit results were taken into account together with the SEI CMM model requirements, ISO 9001 requirements and ISO 9000-3 guidance. The outcome was the definition of primary goals for measurement. In this phase managers were involved both from the baseline project and from other projects as well, representing the viewpoint of the whole software development centre. This activity was coordinated by a metrics promoter together with the external AMI consultant.

The second phase, concerns the analysis of the primary goals to arrive at sub-goals and relevant metrics. This was formalised as a goals tree with corresponding questions leading to the metrics. This activity was generally carried out by the metrics promoter, the AMI consultant, the project leader, the quality co-ordinator and other experts as necessary.

The third phase, concerns the actual metrication, with the introduction of a measurement plan, collection and verification of primitive data processed into measurement data. These tasks were initiated and co-ordinated by the metrics promoter with the participation of project personnel.

The fourth phase concerns managing the improvement resulting from the implementation of the measurement plan. Goals and questions were used to evaluate the measured data and implement corrections or modifications when necessary. Different sets of data were correlated to validate underlying hypotheses. When goals are met, then an improvement goal is achieved and further improvements are possible by repeating the cycle as already indicated in the AMI method description, above.

In parallel to performing the fourth phase - focused always on the baseline project - the overall evaluation of the degree of success of the experiment takes place. All specific data stemming from the AMI pilot implementation are utilised for the evaluation. During this phase, data are also gradually becoming available from other AMI/GQM implementations, following roughly the same phases. In parallel to the fourth phase, information dissemination takes place and expertise is transferred to other areas of application, as well as in other departments involved in software development.

\section{CURRENT STATUS OF THE EXPERIMENT}

The PITA project started in January ' 96 and will conclude in early ' 97 . As a first step in the AMI loop, the software process in the SWDC was assessed, based on the CMM model. CMM was chosen for the following reasons:

- The recognition of CMM as a reference model by many industrial companies and particularly by Ericsson, INTRACOM's biggest customer of software products.

- $\quad \mathrm{CMM}$ assessment results are considered as a credible picture, indicating priorities and general recommendations, as well as being a reusable reference in the future.

- CMM is highly compatible both with general INTRACOM's SWDC improvement efforts and the AMI approach.

After the CMM assessment, findings were checked with baseline project members and used as a first source of information. Additionally, several other sources of goals were explored, such as Policy Deployment initiative results. 
Following the AMI method, a goal tree was constructed, aiming at a better development process, but taking advantage of previous experiment results and integrating different points of view (see Table 1). This tree, reflects project and SWDC concerns about simultaneous search of quality and repeatability in planning and reduced time-to-market. As a first attempt, it was deliberately kept simple, while the possibility exists to integrate other goals later.

Table 1 The PITA experiment Goal Tree for the baseline project

- Improve meeting of internal $\&$ final delivery date with adherence to process

$\Rightarrow$ Improve preparation and maintenance of the sub-project plan

$\Rightarrow$ Decrease impact of early phases results (delays, quality) to the later ones

$\Rightarrow$ Improve project monitoring

- Improve quality as experienced by the customer

$\Rightarrow$ Improve effectiveness of inspection and review

$\Rightarrow$ Improve function test to prevent faults slipping through

$\Rightarrow$ Improve effectiveness of design

$\Rightarrow$ Efficient, adequate Technical Co-ordination

$\Rightarrow$ Increase competence of people

Time-to-market is a key element of SWDC competitiveness and is expressed here through delivery dates. This goal has been expressed as improving achievement of delivery dates both internally and externally. At the same time the processes should be kept coherent..

To reach this goal, two major areas of assessment have been addressed simultaneously: planning and tracking. Since achievement of delivery dates is also the result of the whole project life-cycle, the early phases shall be taken into account as real factors, even if in some cases they are not directly controllable. This is, for instance, the case with requirements related to the baseline project which are handled outside INTRACOM. The impact of early phases is therefore taken as a control factor which cannot be directly improved (being out of the scope of the baseline project), but which is measured and monitored. As shown in the assessment report, higher accuracy in estimation and planning result in increased plan reliability, ability for efficient use of resources and better co-operation.

Improving quality is another concern of the current business of SWDC as a result of the intensive competition inside the telecommunications domain. Quality shall be measured based on customer's perception and at regular check-points. At such intermediate points, the measurements / indicators that were introduced reflect several points of view (quality coordination, test, design, technical co-ordination). The selected factors should affect positively both the quality directly built into the product (due to design process and personnel competence), as well as the activities that reduce defects (testing, inspection). 
This work was documented following the general track of AMI, based on internally used, as well as new, AMI documentation templates or information. An example of tabular representation of a particular goal and breakdown is given in Table 2 .

Table 2 Tabular representation of the Goal: 'Decrease impact of early phases results to the later ones'

\begin{tabular}{|r|l|l}
\hline Analyse & early phases & OBJECT \\
PURPOSE \\
for the purpose of & decreasing & QUALITY FOCUS \\
\hline with respect to & impact & VIEWPOINT \\
\hline from the viewpoint of & project team & ENVIRONMENT \\
\hline in the environment of & FMp4.1 Project &
\end{tabular}

\begin{tabular}{|c|c|c|}
\hline $\begin{array}{l}\text { Quality Focus } \\
\begin{array}{l}\text { Rework effort due to } \\
\text { early phase impact }\end{array}\end{array}$ & $\begin{array}{l}\text { Factors Impacting on Quality Focus } \\
\text { A. requirements stability } \\
\text { B. late events and reviews } \\
\text { C. open technical issues }\end{array}$ & $\begin{array}{l}\text { Change/ Control } \\
\text { A. control } \\
\text { B. control } \\
\text { C. control }\end{array}$ \\
\hline $\begin{array}{l}\text { Baseline/Current/ } \\
\frac{\text { Estimated Quality Focus }}{1 .<\text { current value of }} \\
\text { indicator }> \\
\text { Expected Quality Focus } \\
1 .<\text { indicator target value }>\end{array}$ & \multicolumn{2}{|c|}{$\begin{array}{l}\text { How are Factors Impacting on Quality Focus? } \\
\text { (Note: }+/-=\text { positive/negative trend) } \\
\text { A. the less requirements change, the less project is impacted } \rightarrow-T \\
\text { B. delaying events can delay decision and lead to more rework } \rightarrow- \\
\text { T } \\
\text { open issues can force to rework }->-T\end{array}$} \\
\hline
\end{tabular}

After this step, a measurement plan was set-up including a measurements list (as a table), mechanisms for data collection, roles and responsibilities and a detailed list of possible attributes for measurement description as shown in Table 3.

Table 3 Measurement description attributes

\begin{tabular}{|ll|}
\hline Name & (same as those in a summary and quick reference table) \\
Goal: & remind goal as a textual information \\
Collection procedure: & how the metric is collected, if more information is required \\
& (form and frequencies are defined in the table) \\
Presentation: & the way data are shown, with practical examples \\
Analysis procedure: & how the metric is used, how trends ( $+/$-) are analysed \\
Responsibilities: & for collection, for analysis \\
\hline
\end{tabular}

Regarding the effort spent for the above tasks, the CMM assessment was performed during one week (excluding preparation and training phase for CMM assessment), it involved approximately 50 people from all the levels of SWDC and 5 full-time team members. Goal tree derivation and documentation up to the measurement plan has involved partially 7 persons, and took approximately 4 months. Support for ongoing metrication is 
provided by one person on a part-time basis while other involved personnel participated at scheduled events.

As a follow up of the performance of the CMM assessment and the work started with the AMI metrication, a Software Engineering Process Group (SEPG), as prescribed in the CMM approach, was established in late 1996 to systematically promote and coordinate the various SPI activities at the software design centre. An important part of its activities concerns the systematic implementation of metrics to both SPI activities and ongoing development project work, based on the AMI experience. Actually the approach piloted in the baseline project is being transferred and introduced gradually as a standard process to all new software development projects undertaken.

As one of the key actions of the SEPG, a simplified CMM process assessment was recently performed based on an approach initially introduced in MOTOROLA (Daskalantonakis, 1994) which provided a measured baseline for the software development process capability to plan and track necessary improvements on the CMM ladder. AMI is used to take advantage of results of this baselining at the organisational level to help introduce and guide improvements at both the individual project and organisational levels.

Currently the project is going through its final phases where results are evaluated and further plans established based on the experience acquired to date. Internal dissemination to other areas of software development is also implemented.

\section{EXPERIENCES AND LESSONS FROM THE EXPERIMENT}

During this exercise and based on experiences and results obtained up to this point, some issues proved to be of particular importance and lessons were learned thereof. Some important points are summarised next.

\subsection{Technological point of view}

\section{Usability of Metrics}

Measurements had been introduced at the SWDC, in the past, to address various ad hoc concerns in implementing improvement initiatives. However, their value to the software developers was limited due to the fact that these measurements were mainly reflecting the viewpoint and the objectives of higher management only. On the contrary, the GQM approach and AMI are both based on the integration of various viewpoints (during Goal Analysis), while the supporting measurements provide useful information to all those viewpoints involved and especially the software development practitioners and the software project managers.

AMI provides process visibility to the software development team carrying out a project, by adopting goals that can be tracked through measurement data which are fed back to the development team periodically, during the project progress. In this way, the AMI method enhances motivation and buy-in of the metrication initiative.

Long term measurements are necessary to provide conclusive evidence about product quality and overall development process effectiveness / efficiency. At the same time, shortterm indicators and estimates are necessary to support project planning and monitoring (this 
is especially necessary in the case of long development cycles). Such indicators support an overall (or per development phase) process performance evaluation and decision making.

\section{AMI helps as a backbone of SPI}

AMI is an especially strong aid to plan, perform, monitor and support SPI activities. Particularly important, is the linking of GQM analysis to the CMM paradigm and CMM assessment results. This provides a consolidated and consistent framework of short -and longer- term improvements. In the case of rather immature processes (as it is the case with the PITA baseline project) and where the use of measurements is recent or just beginning, it is necessary to approach SPI by adopting mainly 'knowledge' goals rather than 'change' goals. According to GQM, 'knowledge' goals focus on establishing the current behaviour of used processes while 'change' goals focus on the improvement of the used process. In general, PITA has generated great interest in using AMI/GQM to support current and future SPI activities, resulting in GQM plans being generated in the context of particular SPI activities.

\section{Reuse of existing goals and measurements}

In applying AMI, a crucial step was the identification and collection of all existing goals and metrics (from past or ongoing SPI activities). Such information can and should be reused when establishing the Goal Tree, to reduce overhead and improve consistency with overall operations, policies and targets. In addition, reuse of existing goals and measurements, reduces resistance and provides additional buy-in of the new approach.

\section{Validity of GQM analysis}

INTRACOM's limited experience with Goals and Metrics prior to PITA, causes some concerns that have to be addressed during AMI implementation. Special attention is paid to the analysis and validation of results, for a relatively stable and reliable process measurement baseline to be established. In reality, where there is yet no statistically significant and systematic historical data available, one should avoid quick conclusions and judgements from analysing GQM data.

In such premature situations, it is difficult to establish acceptable measurement ranges. Tentative hypotheses can be made, in order to provide some guidance, but they require validation and review when subsequent conclusive data become available. In the case of some particular goals (e.g. improved design, better project planning), several repetitions / cycles of AMI application, at different projects, may be necessary to obtain a validated GQM model. 


\subsection{Business point of view}

\section{Justification of AMI introduction}

The initial effort expended for PITA establishment, AMI training, assessment, Goal-tree Analysis and Metrication represented a considerable overhead which could not be justified only within the context of a small-to-medium size project ( less than 10 man-years), as it is the case with the baseline project. The overhead from introducing AMI was justifiable only in a wider organisational context, based on the prospect of introducing AMI/GQM gradually to every new SWDC project. The decision of introducing GQM at SWDC had been taken prior to PITA, but PITA itself accelerated the implementation of that decision significantly.

\section{Expansion of use of $A M I / G Q M$}

The AMI/GQM experiences acquired through PITA, should be reused and adapted to an increasing number of other areas (new projects and SPI initiatives). Acceptance and support of $\mathrm{AMI} / \mathrm{GQM}$ has to be ensured at each stage before proceeding further. Expanded use and acceptance of AMI/GQM enhances the impact in the original application area which will be viewed as a pioneering effort.

\section{Development project overheads}

The project overheads caused by a first AMI implementation, based on up to date data is in the order of $4-5 \%$ excluding initial training and CMM assessment. It is expected that this overhead drops significantly in future AMI cycles. Actually, it is expected that when the AMI method gets institutionalised (adopted for all projects, including SPI initiatives), the overall overhead (initiation cycle and measurements feedback loop) will remain low, at a level of around $3 \%$ or even less if automation in data collection can be provided. It is expected to decrease further with the maturing of the metrication process itself (due to reuse of experience etc.). In any case, this overhead is much less than the one associated to bottom-up metrics approaches, which are based on collecting and analysing vast amounts of data. Such data in general concur with experiences reported by other AMI users, from both the original trials of the method, as well as more recent implemetations (Pulford, 1996)

\section{Provision of services}

Outside interest has been expressed for INTRACOM's PITA related experiences and there is potential for INTRACOM to provide metrics and SPI related services to the Greek market of software developers. PITA related experiences and practices should be carefully and gradually transferred in the environment of different organisations. This is because of potential differences in technical and business characteristics, as well as in the case of a less mature software development process. 


\subsection{Strengths and weaknesses of the AMI/GQM approach}

A number of positive comments can be made on the approach followed by PITA. These can be summarised as follows:

- AMI provided a framework for systematic introduction of GQM metrics in INTRACOM. One particular strength was the fact that AMI was found to enhance systematic assessments (CMM-based in our case), whereby the exact status of maturity of the organisation is identified and clear SPI goals are established.

- The Key Process Areas (KPAs) where weaknesses were identified during the assessment will need to be upgraded and become more mature. This process is exceptionally well supported by the GQM metrics. Therefore, in more general terms, a CMM-based SPI activity appears to be strongly supported and enhanced through AMI/GQM. The same applies to other, SPI initiatives (e.g. Policy Deployment) which, through GQM metrics, obtain extra impetus, better monitoring and support.

- The guidance of the training / consulting firm which was contracted for PITA, was a key factor in carrying out the experiment. This is due to the consultant's extensive previous involvement with both the CMM and the AMI. In general, it appears to be of great importance to utilise the appropriate expertise and know-how for initiating SPI activities.

- It is expected that the SWDC will gradually adopt the GQM practices pioneered in PITA, with the necessary adaptations and corrections (as these will be identified during the PITA conclusion phase). In new SWDC projects, when the GQM approach will be standardised, better control and improved effectiveness will be achieved in process areas such as Reviews and Inspections.

On the other hand, a number of problems were identified in the approach followed by PITA. These can be summarised as follows:

- AMI introduction to a baseline project has to be performed in a 'discretionary' way, avoiding any potential disruptions. Introducing $\mathrm{AMI} / \mathrm{GQM}$ is a risky activity for a project which is planned and initiated prior to taking into consideration GQM issues. In the case of PITA, for instance, there was no possibility to establish a baseline project dedicated to the PITA experiment. Thus, one of the normally scheduled and planned projects was used.

- The lead-time of the chosen baseline project actually exceeds the duration of the experiment. Thus, the results obtained from the baseline project, at the end of PITA, will not be final (evaluation will be based on available data at that point of time). For final conclusions to be drawn, the GQM-related activity will have to continue until the end of the baseline project.

- The PITA baseline project receives relatively little benefit from introducing AMI/GQM. However, it is expected that the benefit will be significant for future projects where AMI introduction will be considered from the start (even in the feasibility phase of the project). 
As a result, $A M I / G Q M$ activity will be integrated in a much better way with project planning. On the other hand, since there is a trend for project lead times to become shorter, future AMI evaluation cycles are also expected to become shorter. This last improvement goal could be another AMI/GQM potential application area.

- Another difficulty which was encountered when introducing AMI/GQM as an SPI experiment, stems from the fact that, at INTRACOM no 'measurement baseline' was available (a 'measurement baseline' is a set of measurements on current performance providing a level of reference). As a result, improvement goals cannot be easily quantified (except for high-level management and long-term goals). Initially, only 'knowledge' goals can be used while the gradual creation of a stable performance baseline for GQM goals (and sub-goals) will also allow the introduction of pure 'change' goals.

\section{CONCLUSIONS AND FURTHER ACTIVITIES}

As the PITA project progresses, results from the baseline project are being validated and analysed. Based on such analysis, specific corrections or further improvements to development processes are proposed. At the end of the project, an evaluation of the derived GQM plan (model) will be effected, in order to validate its correctness and to modify it, if and where needed.

An overall evaluation of the effectiveness and benefits of the experiment will be done based on final data. The experience will be packaged, so that the approach with all necessary improvements will be available for reuse within INTRACOM. Already, goal tree analysis and metrication based on PITA experience are being introduced widely (to all new projects) to support specific improvement goals, e.g. improving the effectiveness of SW inspections, as well as to support improvements planned to improve CMM organisational and project capability. In other words, AMI/GQM is becoming institutionalised.

A significant effort is planned and effected in dissemination activities, both internal to INTRACOM and external, for interested developers both in Greek and European markets. Internally, PITA is planned and being executed in such a way, so as to ensure maximum transferability. One important provision was the performance of a SWDC-wide CMM assessment, setting a context for improvements in the whole SWDC. The baseline project AMI application, is largely based on the assessment outcome and the GQM plan (model) derived should be easily adaptable for other projects. Also the CMM/AMI approach will be followed in other areas of software development in INTRACOM.

A new full CMM assessment will also take place later in '97 to mark progress and identify further necessary improvements, starting a whole new cycle of AMI implementation, while 'light' assessments will monitor the situation each semester. Other goals identified in various SPI activities are also to be analysed and measured with AMI. In particular, the AMI approach will also be used to measure the progress and the effectiveness of another EU funded ESSI PIE experiment to be performed at INTRACOM, called PIBOP ('Process Improvement through PSP') - introducing Watt Humphrey's newest initiative, the 
PSP (Personal Software Process) (Humphrey, 1995). This project starts in March '97 and will combine AMI approach with the proposed PSP metrics.

Externally, various dissemination activities presenting the PITA approach and results are scheduled. These include participation in conferences, workshops and seminars, including events organised by the AMI Users Group (AMI, 1993), publications in magazines, newsletters and the Internet (WEB page) as well as best practice dissemination events with INTRACOM cooperating companies. External dissemination is supported by the fact that the approach is quite generic and is easily and largely replicable/adaptable for a wide range of software developers.

\section{REFERENCES}

AMI (1993) WWW-page: http://www.sbu.ac.uk/csse/ami.html

Basili, V.R., Caldiera, G. and Rombach, H.D. (1994), The Goal Question Metric Approach, in Encyclopedia of Software Engineering volume 1, John Wiley \& Sons, pp. 528-532, ISBN 0-471-54004-8.

Boehm, B.W. (1991), Software Risk management: principles and practices, IEEE Software, Jan. '91, pp. 32-41.

Brooks, F. P. (1995), The Mythical Man-Month, Anniversary ed., Addison Wesley, ISBN 0201-83595-9.

Carnegie Mellon University / Software Engineering Instute - CMU/SEI (1995) The Capability Maturity Model: Guidelines for Improving the Software Process, AddisonWesley Publishing Company, ISBN 0-201-54664-7.

Daskalantonakis M.K. (1994), Achieving Higher SEI Levels, IEEE Software, July 1994, pp 17-24.

Dymond, K.M. (1995), A guide to CMM, Process Inc. US, ISBN 0-9646008-0-3.

Fenton, N.E. (1991), Software Metrics, A rigorous approach, Chapman and Hall, London, ISBN 0-412-40440-0 [The new edition to be published in early 1997, written jointly with Pfleeger S.H].

Grady, R.B. and Caswell, D.L. (1987), Software Metrics: Establishing a company-wide program, Prentice Hall, ISBN 0-13-821844-7.

Grady, R.B. (1992), Practical Software Metrics for Project Management and Process Improvement, Prentice Hall, 1992, ISBN 0-13-720384 -5.

Gresse C., Hoisl B and Wust J. (1995), A Process Model for GQM-Based Measurement, Kaiserslautern University (Postfach 3049 D-67653, Kaiserslautern, Germany), STTIReport STTI-95-04-E.

Humphrey, W.S. (1989), Managing the software process, Addison-Wesley Publishing Company, ISBN 0-201-18095-2.

Humphrey, W.S. (1995), A discipline for software engineering, Addison-Wesley Publishing Company, ISBN 0-201-54610-8.

Kan, S.H. (1995), Metrics and models in software quality engineering, Addison-Wesley Publishing Company, ISBN 0-201-63339-6

Matsubara, T. (1991), Project Management in Japan, American Programmer, June 1991, pp. 41-50. 
METKIT (1992) Software Engineering Measurement Teaching Materials. Information is available on Web page URL : http://www.sbu.ac.uk/csse/metkit.html.

Moeuller, K.H. and Paulish, D.J. (1992), Software Metrics: A Practitioner's Guide to Improved Product Development, Chapman \& Hall ISBN 0-412-45900-0.

Pulford K., Kuntzmann-Combelles, A. and Shirlaw S., (1996) A quantitative approach to Software Management - The ami Handbook, Addison-Wesley Publishing Company, ISBN 0-201-87746-5.

Sheridan, B.M. (1993), Policy Deployment, The TQM approach to long-range planning, ASQC Quality Press, ISBN 0-87389-129-5.

\section{BIOGRAPHIES}

Vassilios Sylaidis is managing the software quality assurance section at INTRACOM S.A. Besides overseeing quality assurance activities in the Software Design Centre and other departments developing software, he coordinates various software process improvement activities and programmes at the Software Design Centre. Prior to joining INTRACOM, Sylardis worked as Special Scientist in R\&D (combat systems software development) in the Hellenic Ministry of Defence. Sylaidis received an Electr./Mech. Engineer Diploma from the National Technical University of Athens (NTUA), Greece and a M.Sc. in computer science from the George Washington University, Washington D.C., USA. He is a member of the Hellenic Quality Forum.

Ioannis Nanakis is a senior software quality assurance engineer at INTRACOM S.A. Software Design Centre. He is responsible for software quality methods, software process assessment and improvement, and for the maintenance of the Quality System at the Software Design Centre. He is member of the CMM Assessors Network within the Ericsson Group, and of the Software Engineering Process Group at the Software Design Centre. Nanakis received a Diploma in electrical \& electronics engineering from the National Technical University of Athens (NTUA) and an M.Sc. in systems \& control from the University of Manchester Institute of Science \& Technology (UMIST), UK.

Vassilis Kopanas is a project coordinator at INTRACOM's Development Programmes Department. Since 1991 he is responsible for the management of INTRACOM's CEU funded projects in the area of software engineering and knowledge engineering. He holds a Diploma in electrical \& electronics engineering from the National Technical University of Athens, Greece. He also received an M.Sc. and a Ph.D. in Computer Science from the University of Manchester Institute of Science \& Technology (UMIST), UK. 